\title{
MultiOpED: \\ A Corpus of Multi-Perspective News Editorials
}

\author{
Siyi Liu \\ Sihao Chen Xander Uyttendaele \\ University of Pennsylvania \\ \{siyiliu, sihaoc, xanderu, danroth\}@seas.upenn.edu
}

\begin{abstract}
We propose MultiOpED ${ }^{1}$, an open-domain news editorial corpus that supports various tasks pertaining to the argumentation structure in news editorials, focusing on automatic perspective discovery. News editorial is a genre of persuasive text, where the argumentation structure is usually implicit. However, the arguments presented in an editorial typically center around a concise, focused thesis, which we refer to as their perspective. MULTIOPED aims at supporting the study of multiple tasks relevant to automatic perspective discovery, where a system is expected to produce a singlesentence thesis statement summarizing the arguments presented. We argue that identifying and abstracting such natural language perspectives from editorials is a crucial step toward studying the implicit argumentation structure in news editorials.

We first discuss the challenges and define a few conceptual tasks towards our goal. To demonstrate the utility of MULTIOPED and the induced tasks, we study the problem of perspective summarization in a multi-task learning setting, as a case study. We show that, with the induced tasks as auxiliary tasks, we can improve the quality of the perspective summary generated. We hope that MULTIOPED will be a useful resource for future studies on argumentation in the news editorial domain.
\end{abstract}

\section{Introduction}

News editorial is a form of persuasive text that conveys consensus opinion on a controversial topic from the editors of a newspaper. Much like an argumentative essay, a news editorial centers around a thesis, which represents the authors' perspective on the topic. Usually, a news editorial argues in favor of the authors' stance on the topic, and is substantiated by extensive factual evidence.

\footnotetext{
${ }^{1}$ The authors would like to thank Daniel Ravner, the CEO of www.theperspective.com, for granting access to data from the site for academic research.
}

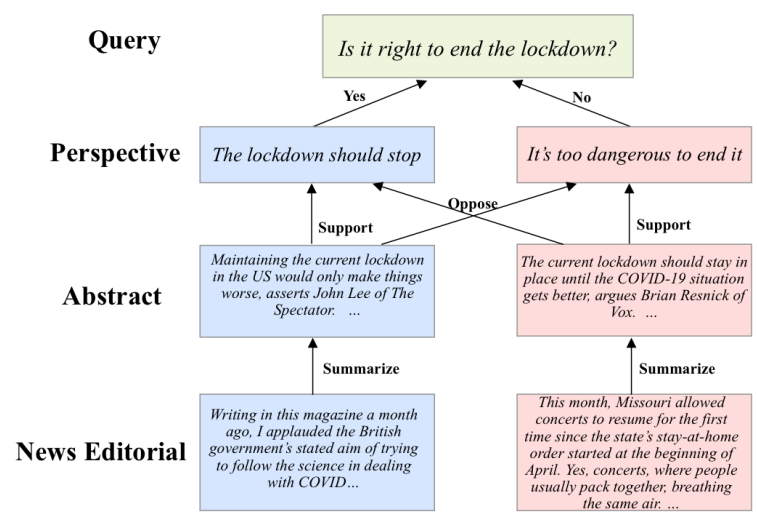

Figure 1: Structure of MULTIOpED. For each query on a controversial topic, two (rather long) news editorials respond to the query from different point-of-views. Each editorial comes with a single paragraph abstract plus a one-sentence perspective, that abstractively summarizes the editorial's key argument in the context of the query. The two resulting perspectives serve as responses with opposite stance to the query.

As news editorials function as professionally produced written discourse for conveying media attitude and guidance, they have traditionally been studied by the community as a rich resource for many argumantation-related tasks. (Wilson and Wiebe, 2003; Yu and Hatzivassiloglou, 2003; Bal and Saint-Dizier, 2009).

This work targets the problem of developing computational methods to identify and comparatively analyze the authors' perspectives and supporting arguments behind news editorials. One challenge to studying the argumentation structure in news editorials is that its elements are rarely expressed explicitly (El Baff et al., 2018). For example, Figure 1 shows two news editorials holding opposite views on whether a lockdown should continue. However, neither of them present their key perspectives explicitly. Instead, the perspective is conveyed through subtle rhetoric strategies to either affirm or challenge the readers' stance from prior belief on the topic, as a study by El Baff 
et al. (2018) discovers. As Figure 1 shows, the statement "The lock down should stop" concisely summarizes the perspective expressed in the article on the left. We refer to such statements as "perspectives" throughout the paper. The ability to abstractively summarize the perspectives from the editorial would allow us to understand multiple topic-aligned editorials in context and reason about their inter-editorial argumentation structure.

To facilitate research along the line, we collect data from THEPERSPECTIVE ${ }^{2}$ website, and construct MultiOpED, an open-domain English news editorial corpus that supports various tasks pertaining to the argumentation structure in news editorials, focusing on automatic perspective discovery (Chen et al., 2019). The structure of the data is shown in Figure 1. For each of the 1,397 natural language query on a different topic in our dataset, it features two (rather long) news editorials. Each editorial features a single-sentence perspective, which is abstractively summarized from the editorial by human experts. A short abstract then highlights the details in the editorial that support the perspective. The perspectives of the two editorials represents responses of opposite stances towards the query.

Naturally, the structure of the dataset induces a range of important argumentation-related natural language understanding tasks. For instance, the presence of the summary perspective allows for stance classification (Hasan and $\mathrm{Ng}, 2013$ ) with respect to the query, which arguably is more tangible than inferring the stance from the entire editorial. Another example task is the conditional generation of the perspective from the abstract/editorial, which relates to the widely studied task of argument generation (Hua and Wang, 2018; Alshomary et al., 2020). We defer the more detailed description of the induced tasks to Section 3.

One key advantage of MULTIOPED that is absent from earlier datasets is that a large number of argumentation-related tasks can be studied jointly using a single high quality corpus. To demonstrate this benefit and the utility of the MultiOpED dataset ${ }^{3}$ along with its induced tasks, we study the problem of perspective summarization in a multitask learning setting. We employ perspective relevance and stance classifications as two auxilliary tasks to the summarization objective. Our empiri-

\footnotetext{
${ }^{2}$ https: //www. theperspective.com/ perspectives/

${ }^{3}$ Our code and data is available at http:// cogcomp. org/page/publication_view/935
}

cal and human analysis on the generated summaries show that the multi-task learning setting improves the generated perspectives in terms of the argument quality and stance consistency.

In summary, our contributions in this work are three-fold. First, we propose a conceptual framework for identifying and abstracting the perspectives and the corresponding argumentation structure in news editorials, and define a set of tasks necessary for achieving this goal. Second, we propose the MultiOpED dataset, a news editorial dataset that induces multiple argumentation-related tasks. Third, we demonstrate the utility of our multi-purpose dataset and induced tasks, by using the perspective summarization task as a case study. We include the induced tasks as auxiliary objectives in multi-task learning setting, and demonstrate their effectiveness to perspective summarization.

\section{Design Principles}

Our goal of perspective discovery follows similar definition proposed by Chen et al. (2019), and is closely related to a widely studied area of argumentation mining, i.e. identifying the argumentation structure within persuasive text (Stab and Gurevych, 2014b; Kiesel et al., 2015). However, most studies in this domain focus on extractive methods, which becomes less applicable to our study. As the arguments are usually presented in an subtle and implicit way in news editorials, we instead focus on the generation methods for the perspectives. This closely resembles the argument conclusion generation task (Alshomary et al., 2020). One key distinction here is the presense of query to provide topic guidance during the perspective generation.

Compared to other conditional text generation tasks, perspective generation subjects to a few more constraints with respect to the argumentation structure. For example, the perspective must constitute the same stance (Hasan and Ng, 2013) as the editorial towards the query. On the other hand, while the editorial may cover content not directly related to the query, the generated perspective must present a relevant argument in the query's context. Such structural constraints can be studied in the format of classification problems. And being able to study such problems along side the perspective summarization task on one high-quality corpus is important in our case, as it opens up the probability of modeling the tasks jointly. We show the benefit of 


\begin{tabular}{l|cccc} 
Dataset & Source & Open Domain & Cross Article & Abstractive \\
\hline \hline ARAUCARIADB (Reed et al., 2008) & News Ed. & $\checkmark$ & $\times$ & $\times$ \\
(Stab and Gurevych, 2014a) & Essay & $\checkmark$ & $\times$ & $\times$ \\
(Eckle-Kohler et al., 2015) & News & $\checkmark$ & $\times$ & $\times$ \\
(Hua and Wang, 2018) & Reddit/Wiki. & Politics only & $\times$ & $\checkmark$ \\
PERSPECTRUM (Chen et al., 2019) & Debate & $\checkmark$ & $\times$ & $\checkmark$ \\
\hline MultiOpED & News Ed. & $\checkmark$ & $\checkmark$ & $\checkmark$ \\
\hline
\end{tabular}

Table 1: A comparison across datasets with similar purpose to MulTiOpED. We compare the datasets along three dimensions. Open Domain: whether the dataset features a wide variety of topics. Cross Article: whether the argumentation structure between documents are annotated. Abstractive: whether the elements in argumentation structure is abstractive or extractive.

doing so by presenting a case study in section 5 .

As the query provides topic guidance, it allows for the study of the topic-aligned pairs of editorials which presents counter-arguments to each other. Such property is absent from notable datasets of similar purposes to ours, as shown in Table 1. ARAUCARIADB (Reed et al., 2008) is the first effort to provide large-scale annotations of dense argumentation structure within individual news editorials. Stab and Gurevych (2014a); Eckle-Kohler et al. (2015) provide resources for extractive argumentation structure in persuasive essays and news articles, respectively. Later works (Hua and Wang, 2018; Chen et al., 2019) focus on the abstractive generation or identification of arguments from web corpora. All of these datasets focus on studies of argumentation structure within individual document. Instead, our proposed dataset presents the opportunity to study the cross-document argumentation structure.

\begin{tabular}{lcccc}
\hline Instance & Size & Avg. Len. & Min & Max \\
\hline \hline Query & 1,397 & 7.4 & 3 & 15 \\
Perspective & 2,794 & 6.1 & 2 & 10 \\
Abstract & 2,794 & 101.9 & 47 & 160 \\
Article & 2,584 & 918.6 & 74 & 7,608 \\
\hline
\end{tabular}

Table 2: Statistics of the MultiOpEd dataset. Size represents the number of each valid instance, Avg. Len. indicates the average length of each instance in terms of the number of tokens split by space, and Min and Max represent the number of tokens of the shortest and longest texts of each instance.

\section{MultiOpED and Induced Tasks}

Following the design principles outlined in the previous section, we propose a topic-aligned English news editorial corpus, MultiOpED. The structure of an example instance in MuLTiOpED is shown in Figure 1. To clarify our description of the dataset, we use the following notations. Let $q$ be a query about a controversial topic. Each $q$ in the dataset is paired with two editorials $e_{\text {pro }}$ and $e_{\text {con }}$, that constitute supporting and opposing stances to the query $q$ respectively. Each editorial is abstracted into and a single-sentence perspective $p$, which provides a high-level summarization of the key argument presented in the editorial. The premises, or relevant details to support the perspective, forms the abstract $a$.

Naturally, the relation between these elements induces several tasks, most of which encompass similar definitions to existing argumentation-related tasks. We define and describe the tasks and their connection to our end goal of perspective discovery below.

1. Generating an Abstract: Given an editorial $e$, a system is expected to identify and summarize the relevant arguments into an abstract paragraph $a$ to the context provided by the query $q$. This is closely related to the task of argument synthesis (El Baff et al., 2019; Hua et al., 2019). We set aside this problem in our case study in section 5 , and use the abstract provided by the dataset.

2. Perspective Summarization: Given the generated abstract $a$ and the query $q$, a system is expected to generate the perspective $p$, a concise summary of the arguments presented in $a$. Conceptually, this problem resembles the task of argument conclusion generation (Alshomary et al., 2020). We adopt a slightly different setting where the target topic is expressed in the form of a natural language query.

3. Stance Classification: Our goal is to infer the 


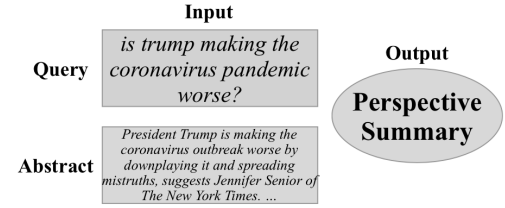

Figure 2: Perspective Summarization: Generate an single sentence argument that represents the key perspective expressed in the news editorial.

editorials $e$ 's stance towards a query $q$. The generated perspective $p$ from editorial $e$ allows us to focus on a simpler task definition of classifying the stance of the perspective to the query $q$ (Hasan and Ng, 2013; Bar-Haim et al., 2017).

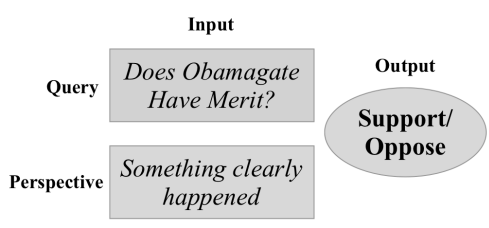

Figure 3: Stance Classification: Decide if the perspective supports or opposes the query.

4. Assessing the Relevance of Perspective: We want to measure the validity of the perspective by assessing whether the perspective presents a relevant argument towards the query (Chen et al., 2019; Ein-Dor et al., 2020). This can be formulated as a classification problem with the query $q$ and a perspective $p$ as inputs, as we show in section 5 .

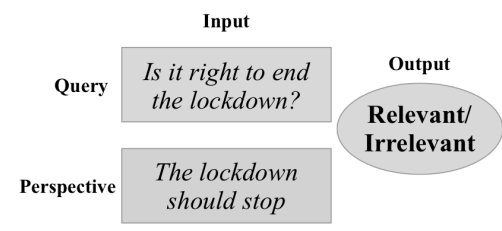

Figure 4: Relevance classification: Decide if the perspective is relevant to the query.

\section{Dataset Construction}

\subsection{Data Collection}

We extract the query, editorial article pairs, abstract paragraph pairs, along with their perspective summaries from THEPERSPECTIVE ${ }^{4}$ website. The website presents controversial topics in the form of queries. For each query, two related editorial articles with opposing views from different sources

\footnotetext{
${ }^{4}$ https: // www. theperspective.com/ perspectives/
}

are selected by the writers from the website. The writers create a concise one-sentence summary of each article as the response to the query, and an abstract paragraph to summarize the relevant arguments from the article. An example structure of the data is shown in Figure.1.

We use BEAUTIFUlSOUP ${ }^{5}$ and NEWSPAPER $3 \mathrm{~K}$ ${ }^{6}$ to extract and clean the perspective and news data.

\subsection{Crowdsource Verification \& Annotation}

To verify the structure from the website, and collect additional annotations, such as stance of the perspectives, we conduct a few annotation experiments with Amazon Mechanical Turk ${ }^{7}$. For all of our annotation experiments, we require the workers to be located in the United States, as the controversial topics covered by the website are most applicable in the U.S. context. We also require the workers to have masters qualifications (i.e. Top performers recognized by MTurk among all workers). We compensate the workers $\$ 0.75, \$ 1.00$ and $\$ 1.25$ per 10 queries for the implicit reference resolution, topic annotation, and stance annotation tasks respectively. The compensation rates are determined by estimating the average completion time for each annotation experiments. Example screenshots of our annotation interface and more detailed annotation guidelines can be found in Appendix B.

\subsubsection{Stance Annotation}

In our dataset, each query is presented with two perspectives with opposite stance to the query. However, the raw data that we collected does not specify the stance of each perspective individually.

We ask two expert annotators label whether each perspective is offering a supporting or opposing view with respect to its query. The two experts discuss and adjudicate their decisions. We then ask on average three crowdsource workers per instance to verify the annotations.

From the annotations collected by experts, we find that 30 out of 1,397 queries do not constitute a clear stance. Such queries are typically "openended" questions which cannot be responded with a yes or no answer, i.e. why or what questions. We leave these instances unlabeled and exclude them from the next verification step.

\footnotetext{
${ }^{5}$ https: / / www . crummy . com/software/ Beautifulsoup/bs4/doc/

${ }^{6}$ https: / / newspaper.readthedocs.io/

${ }^{7}$ https: / / www.mturk.com/
} 
To assess the quality of stance labels created, we randomly sample 500 perspectives, and ask three MTurk workers per instance to verify stance labels. We computed the inter-rater agreement fleiss' $\kappa=0.81$ among workers, and the agreement between majority decision from works and the expert's adjudicated annotations is cohen's $\kappa=0.92$. We describe how we measure the two types of agreements respectively in Appendix A.3.

\subsubsection{Implicit Reference Resolution in Perspectives}

Some of the perspectives in our dataset have implicit references to certain subjects in the query. For instance, for a query "Is Trump Right To Criticize Mail-In Voting?", and a perspective "It's far too risky for an election", the word "It" in the perspective refers to "Mail-in Voting" in the query. As we assume that a perspective should presents a complete, valid argument on itself, we decide to replace such implicit reference in a perspective with the correct referent in the query. For example, the corrected perspective in the previous example would become "Mail-in voting is far too risky for an election".

We ask one expert annotator to identify implicit references and make modifications for every perspective in the dataset. In total, 1,301 out of 2,794 perspectives are identified and corrected by the expert annotator. We ask three Turkers to verify that the modifications do not introduce any grammatical error or change the original meaning. We randomly sample 500 modified perspectives and present Turkers with the question of "Will this modification change the original meaning or introduce grammar error?". The percentage of majority answers being "No" is $84 \%$. We include both changed and original versions of the perspectives in our datasets.

\subsubsection{Topic Annotations}

We create 9 topic labels according to the categorization from THEPERSPECTIVE website and major news outlets. We then as ask three MTurk workers to assign one of the 9 topic labels to each query. We regard the majority answer by the Turkers as the annotation for its topic category. In cases where all three annotators choose different categories (43 cases out of all 1397 queries), we label it as other topics. We show the distribution of topic categories in Figure 5. The inter-agreement among three annotators for this 9-class classification task is $\kappa=0.65$

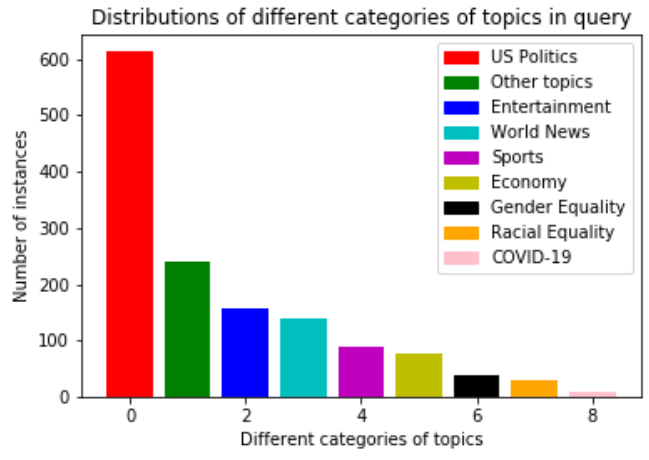

Figure 5: Topic distribution of the 1397 queries in MultiOpEd. Note that the two editorials for each query fall in the same topic category as the query.

\subsection{Dataset Statistics}

MultiOpED consists of 1,397 queries about different news topics. Each query is presented with two perspectives, two abstracts and two linked news editorials. Despite a few stale urls and invalid redirections, we manage to extract the text for 2,584 news editorials. More detailed statistics are reported in Table 2.

\section{Case Study: Multi-task Learning for Perspective Summarization}

\subsection{Multi-Task Framework}

To demonstrate the benefits of modeling the induced tasks on the argumentation structure, we present a case study on the task of perspective summarization. Given a query and an abstract from the related editorial, a system is expected to produce a concise and fluent summary perspective for the editorial. In addition, the generated perspective ideally should satisfy a few structural constraints with respect to the query. For instance, the generated perspective must constitute the same stance as the editorial towards the query. Also the perspective should be relevant in the context of the query. The two requirements resemble the perspective stance and relevance classification" tasks defined in section 3 respectively.

Motivated by this, we study the two tasks together with perspective summarization in a multitask learning framework. We choose BART (Lewis et al., 2020) as our base summarization model. BART is a pretrained auto-regressive transformer (Vaswani et al., 2017) encoder-decoder model, that have been proven effective in conditional text generation and other NLP tasks. 


\begin{tabular}{c|cccc|cc}
\hline Model & ROUGE $_{1}$ & ROUGE $_{2}$ & ROUGE $_{L}$ & BERTSCORE & REL. \% & STANCE \% \\
\hline BART & 28.24 & 11.34 & 26.96 & 88.67 & 91.91 & 72.32 \\
+ Rel & 28.35 & 11.51 & 27.12 & 88.69 & 92.98 & 72.68 \\
+ Stance & 28.19 & 11.53 & 26.93 & $\mathbf{8 8 . 7 5}$ & 91.25 & 73.39 \\
+ Rel \& Stance & $\mathbf{2 9 . 1 8}$ & $\mathbf{1 1 . 9 2}$ & $\mathbf{2 7 . 9 4}$ & 88.74 & $\mathbf{9 4 . 6 4}$ & $\mathbf{7 4 . 2 9}$ \\
\hline
\end{tabular}

Table 3: Results of our multitask perspective summarization models. We compare to BART as a baseline, and experiment with different combinations of the auxiliary tasks. We report the $F_{1}$ score under RoUGE $\{1,2, L\}$ and BERTSCORE metrics, as well as the percentage of summaries with the correct relevance and stance label, as predicted by our pretrained classification models respectively. See Appendix A for training details and hyperparameters settings.

\begin{tabular}{c|cc|cc}
\hline Model & RANK & \%1ST & REL. & STANCE \\
\hline BART & 2.09 & 49.50 & 77.00 & 70.50 \\
+ Rel & $\mathbf{1 . 7 4}$ & $\mathbf{6 0 . 5 0}$ & $\mathbf{8 7 . 0 0}$ & 70.50 \\
+ Stance & 1.78 & 58.50 & 83.00 & $\mathbf{7 9 . 5 0}$ \\
+ R \& $S$ & 1.76 & 59.00 & 82.00 & 69.00 \\
\hline
\end{tabular}

stance models achieve binary accuracy of $92 \%$ and $75 \%$ respectively.

During the perspective summarization model training, we use the pre-trained BERT models for relevance and stance classification to predict labels for each generated summary. We expect the BART

Table 4: Human Evaluations results. "RANK" shows a model's averaged rank judged by the raters $(1=$ best, 4 =worst) " $\% 1 \mathrm{sT}$ " represents the percentage of generated summaries from one model that are ranked the best. We allow ties in the ranking. REL. and STANCE are the percentages of generated summaries that are relevant to and have the correct stance with respect to the query.

We start with a pretrained BART base model with $139 M$ parameters, and finetune the model to output the target perspective given the query and abstract concatenated as input. In addition, we put two separate linear layers over the pooled embeddings of the last decoder layer, and predict the relevance and stance labels of the generated summary respectively. The two tasks and the perspective summarization are learned jointly, and share the underlying model parameters from BART.

One obvious challenge in the setup is that we do not have access to the ground truth stance and relevance labels for the generated summaries during training. To address this, we adopt similar strategies as in knowledge distillation (Hinton et al., 2015). We first train two separate BERT (Devlin et al., 2019) classifiers as the teacher models for stance and relevance classificaiton respectively. Due to the size limit of our dataset, we pretrain both models on the PERSPECTRUM dataset (Chen et al., 2019), which contain over 7,000 instances of training data, with similar formats and definition to our (query, perspective) pairs. We further fine-tune the models on our training set. When measured against our test set, the relevance and plus linear layers to "mimic" the predictions made by the two pretrained BERT models respectively. Specifically:

$$
\begin{aligned}
& \mathcal{H}_{\mathrm{Q}}=\operatorname{EOS}\left(\mathcal{D}_{\mathrm{BART}}\left(\mathcal{E}_{\mathrm{BART}}(\mathcal{Q})\right)\right) \\
& \mathcal{H}_{\mathrm{A}}=\operatorname{EOS}\left(\mathcal{D}_{\mathrm{BART}}\left(\mathcal{E}_{\mathrm{BART}}(\mathcal{A})\right)\right)
\end{aligned}
$$

We feed the query and the abstract separately through the BART encoder ( $\left.\mathcal{E}_{\text {BART }}\right)$ and decoder $\left(\mathcal{D}_{\text {BART }}\right)$. We get their hidden representations $\mathcal{H}_{\mathrm{Q}}$ and $\mathcal{H}_{\mathrm{A}}$ as the embedding of the end-of-sentence $('</ \mathrm{s}>$ ') token from the decoder. We then concatenate $\mathcal{H}_{\mathrm{Q}}$ and $\mathcal{H}_{\mathrm{A}}$, and feed the concatenation to the two linear layers. Finally, a softmax layer is applied to get stance/relevance predictions $\tilde{y}_{r e l}$ and $\tilde{y}_{\text {stance }}$.

$$
\begin{aligned}
\tilde{y}_{\text {stance }} & =\operatorname{SofTMAx}\left(W_{s}^{T}\left[\mathcal{H}_{\mathrm{Q}}, \mathcal{H}_{\mathrm{A}}\right]\right) \\
\tilde{y}_{\text {rel }} & =\operatorname{Softmax}\left(W_{r}^{T}\left[\mathcal{H}_{\mathrm{Q}}, \mathcal{H}_{\mathrm{A}}\right]\right)
\end{aligned}
$$

Next, We feed the query and the generated summary to the two pretrained BERT classification models to get the soft stance and relevance labels $y_{\text {rel }}$ and $y_{\text {stance. }}$. We use two mean square error (MSE) loss terms to measure the discrepancy between the BART predictions and the soft labels.

$$
\begin{aligned}
\mathcal{L}_{\mathrm{REL}} & =\operatorname{MSELOSS}\left(y_{\text {rel }}, \tilde{y}_{\text {rel }}\right) \\
\mathcal{L}_{\mathrm{STANCE}} & =\operatorname{MSELOSS}\left(y_{\text {stance }}, \tilde{y}_{\text {stance }}\right)
\end{aligned}
$$

We combine $\mathcal{L}_{\text {ReL }}$ and $\mathcal{L}_{\text {STANCE }}$ with the summarization objective, $\mathcal{L}_{\text {SUM }}$, which is the negative loglikelihood loss between generated and target perspective. The auxiliary losses $\mathcal{L}_{\mathrm{REL}}$ and $\mathcal{L}_{\mathrm{STANCE}}$ 
are weighted by tunable hyperparameters $\alpha_{1}$ and $\alpha_{2}$ respectively.

$$
\mathcal{L}=\mathcal{L}_{\mathrm{SUM}}+\alpha_{1} \cdot \mathcal{L}_{\mathrm{REL}}+\alpha_{2} \cdot \mathcal{L}_{\mathrm{STANCE}}
$$

\subsection{Results}

\subsubsection{Automatic Evaluations}

Table 3 shows our evaluation results of our multitask model with different combinations of auxiliary tasks. The reported results are averaged over three trained models with different random initialization. We first evaluate the generated perspective summaries against the target perspective with Rouge (Lin, 2004) and BERTSCORE (Zhang et al., 2020) metrics. We observe that relevance and stance auxiliary tasks both increase the ROUGE and BERTSCORE, and combining the two objectives yields the best performance under the ROUGE metrics.

To empirically verify whether the perspectives generated by our multi-task model are improved in terms of the relevance and stance correctness, we again use the two pretrained BERT classifiers to measure the percentage of generated summary with correct relevance and stance label. The results potentially suggest that by "mimicing" the predictions made by the two pretrained classifiers, our multitask framework is able to generate summaries with higher quality along the two dimensions.

\subsection{Human Evaluations}

We randomly sampled 100 instances of abstracts with query from the test set, and ask two human raters to judge the quality of perspectives generated by the four systems. For the four summaries generated from an abstract by the different systems, we shuffle their order and ask the raters to rank each summary by the overall quality, with four criteria considered (1) Fluency (2) Grammatical Correctness (3) Faithfulness to the arguments offered in the original abstract (4) Salience. We allow ties among different summaries. We report their averaged ranks and the number of times a system is ranked first place in Table 4 . The results are the averaged scores between the two annotators, and the level of agreement between them for this 4-class ranking task is $\kappa=0.35$.

For each summary, we ask the raters to annotate whether it (1) represents a relevant argument to the query (2) constitutes the correct stance as the target stance label. The kappa agreement between the two

\begin{tabular}{|c|c|}
\hline Query & $\begin{array}{c}\text { Should trump accept democrats' } \\
\text { gov't spending bill? }\end{array}$ \\
\hline \hline BART & $\begin{array}{c}\text { A shutdown is the best } \\
\text { deal he can get. }\end{array}$ \\
\hline + REL & $\begin{array}{c}\text { Trump should accept } \\
\text { the gop budget. }\end{array}$ \\
\hline \hline Gold & $\begin{array}{c}\text { This deal is the most } \\
\text { achievable compromise. }\end{array}$ \\
\hline
\end{tabular}

Table 5: An example where relevance auxiliary task helps the perspective summarization process

\begin{tabular}{|c|c|}
\hline Query & $\begin{array}{c}\text { Is apple's iphone x technology } \\
\text { any good? }\end{array}$ \\
\hline \hline BART & $\begin{array}{c}\text { Apple's new iPhone X offers } \\
\text { many great opportunities }\end{array}$ \\
\hline + STANCE & $\begin{array}{c}\text { Apple's new face-recognition } \\
\text { technology raises many ethical } \\
\text { issues }\end{array}$ \\
\hline \hline Gold & $\begin{array}{c}\text { Apple's new Iphone X raises } \\
\text { many security concerns }\end{array}$ \\
\hline
\end{tabular}

Table 6: An example where stance auxiliary task helps the perspective summarization process

raters for these two tasks are 0.54 and 0.70 , respectively. We show the human evaluation results in Table 4 . We observe that while both the relevance and stance auxiliary tasks improve the quality of the generated perspective, combining the two auxiliary tasks does not guarantee a better summary quality.

\subsection{Analysis and Discussion}

The results on ROUGE, BERTSCORE and human evaluation suggest that the perspective summarization model learning benefits from both the relevance and stance tasks. However, we also observe that the vanilla BART present a strong baseline in both automatic and human evaluations.

We list two typical cases where we observe the relevance and stance objectives improve the quality of the generated summary. For the query shown in Table 5, the BART model generates an out-ofcontext word "shutdown", which exists in the abstract, but is not applicable in the context provided by the query. The model with relevance objective, on the other hand, generates a perspective that is coherent to the context provided. For the query shown in Table 6, the baseline BART model incorrectly produces a supporting perspective to the 
query, while the editorial or abstract presents the opposite stance. The model with the stance objective generates a perspective with a matching stance.

While we choose relevance and stance classification as the two auxiliary tasks in this case study, there exist many other candidate tasks that might be helpful in the setting. For instance, measuring the quality (Toledo et al., 2019), or more specifically persuasiveness (Carlile et al., 2018) of the perspective might be two, amongst other, viable options. As our study assumes that the abstract is provided for each editorial, the overall performance of perspective summarization will likely drop, if we use model-generated abstract instead of ground truth as input.

\section{Related Work}

\subsection{Argumentation in News Editorials}

News editorials have been studied as a resource for studying many argumentation-related tasks. Wilson and Wiebe (2003); Yu and Hatzivassiloglou (2003) use editorials for the study on sentiments and opinions. Later works (Reed et al., 2008; Bal and Saint-Dizier, 2009; Chow, 2016) shift focus on the argumentation structure within editorials, and their persuasiveness effect ( $\mathrm{Al}$ Khatib et al., 2016; El Baff et al., 2020). A few other recent studies have explored argument quality (El Baff et al., 2018) and generation (El Baff et al., 2019) when using editorials as a resource.

Our proposed dataset and study focus on the interplay between elements of the argumentation structure presented in editorial articles. Unlike previous work, we study these elements as the abstractive instead of extractive summary from the news editorials.

\subsection{Argument Generation}

Most early efforts in argument generation, i.e. generating components in an argumentation structure, study rule-based synthesis methods based on argumentation theories (Reed et al., 1996; Zukerman et al., 2000). With the recent progress in neural, sequence to sequence text generation methods (Sutskever et al., 2014), a few studies have adapted such techniques for end-to-end argument generation. (Wang and Ling, 2016; Hua and Wang, 2018; Hua et al., 2019).

The task of perspective generation in this work closely relates to argument conclusion generation (Alshomary et al., 2020). Our study focuses on the setting where the target topic, or the query, is given as input to the generation model. Due to the implicit nature of the perspectives (Habernal et al., 2018), one key challenge to the task is keep the semantics of the perspective generated truthful to the abstract and editorial article. We approach this by measuring the compatibility of the perspective to the context along the dimensions of content salience (Bar-Haim et al., 2020) and stance correctness (Bar-Haim et al., 2017). Our multi-task generation approach conceptually resembles the work by Guo et al. (2018), where multiple auxiliary tasks is employed to improve the quality of the generated summary.

\section{Conclusion}

We present MultiOpED an open-domain news editorial corpus that induces a number of argumentation-related tasks. The proposed dataset presents a few properties that are absent from existing datasets. First, the elements in the annotation structure are presented as abstraction over the text in editorial, as such elements usually exist implicitly in editorials. Second, as the pairs of editorials are aligned by topic, and exhibit opposing stance to each other, such structure allows for studies on cross-document argumentation structure. Third, the dataset allows for the study of multiple argumentation-related tasks together.

To demonstrate the power of having multiple related tasks in a single high-quality dataset, we study the problem of perspective summarization in a multi-task learning setting. Our analysis shows that modeling stance and relevance classification jointly with the summarization task improves the overall quality of the perspective generated.

In future work, we hope to utilize the corpus to improve the multi-task framework for perspective summarization. As we set aside the problem of abstract generation in our case study, we would also like to identify the challenges and potential solution to the problem. We hope that MULTIOPED presents opportunities and challenges to future research in argumentation.

\section{Ethical Considerations}

We collected data for MULTIOPED by automatically extracting data from www. theperspective. com/perspectives. The CEO of the website, Daniel Ravner, granted us permission to extract and use their data for academic research. We fur- 
ther annotated the data using crowd-workers. All crowd-workers were compensated by a fair wage determined by estimating the average completing time of each annotation task. Please refer to section 4.2 for more details.

The queries, abstracts, and perspectives in MULTIOPED are written by the professional writers of the website. The website aims at presenting the perspectives in each article without unnecessary subjective interpretation, but there is no guarantee that no subjectivity is involved in their content creation process.

\section{Acknowledgments}

The authors would like to thank Daniel Ravner, the CEO of www. theperspective.com, for kindly granting access to data from the site for academic research. This work was supported in part by a Focused Award from Google, and a gift from Tencent.

\section{References}

Khalid Al Khatib, Henning Wachsmuth, Johannes Kiesel, Matthias Hagen, and Benno Stein. 2016. A News Editorial Corpus for Mining Argumentation Strategies. In Proceedings of COLING 2016, the 26th International Conference on Computational Linguistics: Technical Papers, pages 3433-3443.

Milad Alshomary, Shahbaz Syed, Martin Potthast, and Henning Wachsmuth. 2020. Target inference in argument conclusion generation. In Proceedings of the 58th Annual Meeting of the Association for Computational Linguistics, pages 4334-4345, Online. Association for Computational Linguistics.

Bal Krishna Bal and Patrick Saint-Dizier. 2009. Towards an analysis of argumentation structure and the strength of arguments in news editorials. The Society for the Study of Artificial Intelligence and the Simulation of Behaviour.

Roy Bar-Haim, Indrajit Bhattacharya, Francesco Dinuzzo, Amrita Saha, and Noam Slonim. 2017. Stance classification of context-dependent claims. In Proceedings of the 15th Conference of the European Chapter of the Association for Computational Linguistics: Volume 1, Long Papers, pages 251-261, Valencia, Spain. Association for Computational Linguistics.

Roy Bar-Haim, Lilach Eden, Roni Friedman, Yoav Kantor, Dan Lahav, and Noam Slonim. 2020. From arguments to key points: Towards automatic argument summarization. In Proceedings of the 58th Annual Meeting of the Association for Computational Linguistics, pages 4029-4039, Online. Association for Computational Linguistics.
Winston Carlile, Nishant Gurrapadi, Zixuan Ke, and Vincent Ng. 2018. Give me more feedback: Annotating argument persuasiveness and related attributes in student essays. In Proceedings of the 56th Annual Meeting of the Association for Computational Linguistics (Volume 1: Long Papers), pages 621631, Melbourne, Australia. Association for Computational Linguistics.

Sihao Chen, Daniel Khashabi, Wenpeng Yin, Chris Callison-Burch, and Dan Roth. 2019. Seeing Things from a Different Angle: Discovering Diverse Perspectives about Claims. In Proc. of the Annual Conference of the North American Chapter of the Association for Computational Linguistics (NAACL).

Marisa Chow. 2016. Argument identification in Chinese editorials. In Proceedings of the NAACL Student Research Workshop, pages 16-21, San Diego, California. Association for Computational Linguistics.

Jacob Devlin, Ming-Wei Chang, Kenton Lee, and Kristina Toutanova. 2019. BERT: Pre-training of deep bidirectional transformers for language understanding. In Proceedings of the 2019 Conference of the North American Chapter of the Association for Computational Linguistics: Human Language Technologies, Volume 1 (Long and Short Papers), pages 4171-4186, Minneapolis, Minnesota. Association for Computational Linguistics.

Judith Eckle-Kohler, Roland Kluge, and Iryna Gurevych. 2015. On the role of discourse markers for discriminating claims and premises in argumentative discourse. In Proceedings of the 2015 Conference on Empirical Methods in Natural Language Processing, pages 2236-2242, Lisbon, Portugal. Association for Computational Linguistics.

Liat Ein-Dor, Eyal Shnarch, Lena Dankin, Alon Halfon, Benjamin Sznajder, Ariel Gera, Carlos Alzate, Martin Gleize, Leshem Choshen, Yufang Hou, et al. 2020. Corpus wide argument mining-a working solution. In Proceedings of the AAAI Conference on Artificial Intelligence, volume 34, pages 7683-7691.

Roxanne El Baff, Henning Wachsmuth, Khalid Al Khatib, Manfred Stede, and Benno Stein. 2019. Computational argumentation synthesis as a language modeling task. In Proceedings of the 12th International Conference on Natural Language Generation, pages 54-64, Tokyo, Japan. Association for Computational Linguistics.

Roxanne El Baff, Henning Wachsmuth, Khalid AlKhatib, and Benno Stein. 2018. Challenge or empower: Revisiting argumentation quality in a news editorial corpus. In Proceedings of the 22nd Conference on Computational Natural Language Learning, pages 454-464, Brussels, Belgium. Association for Computational Linguistics.

Roxanne El Baff, Henning Wachsmuth, Khalid Al Khatib, and Benno Stein. 2020. Analyzing the 
Persuasive Effect of Style in News Editorial Argumentation. In Proceedings of the 58th Annual Meeting of the Association for Computational Linguistics, pages 3154-3160, Online. Association for Computational Linguistics.

Joseph L. Fleiss and Jacob Cohen. 1973. The equivalence of weighted kappa and the intraclass correlation coefficient as measures of reliability. Educational and Psychological Measurement, 33(3):613619.

Han Guo, Ramakanth Pasunuru, and Mohit Bansal. 2018. Soft layer-specific multi-task summarization with entailment and question generation. In Proceedings of the 56th Annual Meeting of the Association for Computational Linguistics (Volume 1: Long Papers), pages 687-697, Melbourne, Australia. Association for Computational Linguistics.

Ivan Habernal, Henning Wachsmuth, Iryna Gurevych, and Benno Stein. 2018. The argument reasoning comprehension task: Identification and reconstruction of implicit warrants. In Proceedings of the 2018 Conference of the North American Chapter of the Association for Computational Linguistics: Human Language Technologies, Volume 1 (Long Papers), pages 1930-1940.

Kazi Saidul Hasan and Vincent Ng. 2013. Stance classification of ideological debates: Data, models, features, and constraints. In Proceedings of the Sixth International Joint Conference on Natural Language Processing, pages 1348-1356, Nagoya, Japan. Asian Federation of Natural Language Processing.

Geoffrey Hinton, Oriol Vinyals, and Jeffrey Dean. 2015. Distilling the knowledge in a neural network. In NIPS Deep Learning and Representation Learning Workshop.

Xinyu Hua, Zhe Hu, and Lu Wang. 2019. Argument generation with retrieval, planning, and realization. In Proceedings of the 57th Annual Meeting of the Association for Computational Linguistics, pages 2661-2672, Florence, Italy. Association for Computational Linguistics.

Xinyu Hua and Lu Wang. 2018. Neural argument generation augmented with externally retrieved evidence. In Proceedings of the 56th Annual Meeting of the Association for Computational Linguistics (Volume 1: Long Papers), pages 219-230, Melbourne, Australia. Association for Computational Linguistics.

Johannes Kiesel, Khalid Al-Khatib, Matthias Hagen, and Benno Stein. 2015. A shared task on argumentation mining in newspaper editorials. In Proceedings of the 2nd Workshop on Argumentation Mining, pages 35-38, Denver, CO. Association for Computational Linguistics.

Mike Lewis, Yinhan Liu, Naman Goyal, Marjan Ghazvininejad, Abdelrahman Mohamed, Omer
Levy, Veselin Stoyanov, and Luke Zettlemoyer. 2020. BART: Denoising sequence-to-sequence pretraining for natural language generation, translation, and comprehension. In Proceedings of the 58th Annual Meeting of the Association for Computational Linguistics, pages 7871-7880, Online. Association for Computational Linguistics.

Chin-Yew Lin. 2004. ROUGE: A package for automatic evaluation of summaries. In Text Summarization Branches Out, pages 74-81, Barcelona, Spain. Association for Computational Linguistics.

Chris Reed, Derek Long, and Maria Fox. 1996. An architecture for argumentative dialogue planning. In International Conference on Formal and Applied Practical Reasoning, pages 555-566. Springer.

Chris Reed, Raquel Mochales Palau, Glenn Rowe, and Marie-Francine Moens. 2008. Language resources for studying argument. In LREC 2008.

Christian Stab and Iryna Gurevych. 2014a. Annotating argument components and relations in persuasive essays. In Proceedings of COLING 2014, the 25th International Conference on Computational Linguistics: Technical Papers, pages 1501-1510, Dublin, Ireland. Dublin City University and Association for Computational Linguistics.

Christian Stab and Iryna Gurevych. 2014b. Identifying argumentative discourse structures in persuasive essays. In Proceedings of the 2014 Conference on Empirical Methods in Natural Language Processing (EMNLP), pages 46-56, Doha, Qatar. Association for Computational Linguistics.

Ilya Sutskever, Oriol Vinyals, and Quoc V Le. 2014. Sequence to sequence learning with neural networks. In Advances in Neural Information Processing Systems, pages 3104-3112.

Assaf Toledo, Shai Gretz, Edo Cohen-Karlik, Roni Friedman, Elad Venezian, Dan Lahav, Michal Jacovi, Ranit Aharonov, and Noam Slonim. 2019. Automatic argument quality assessment - new datasets and methods. In Proceedings of the 2019 Conference on Empirical Methods in Natural Language Processing and the 9th International Joint Conference on Natural Language Processing (EMNLPIJCNLP), pages 5625-5635, Hong Kong, China. Association for Computational Linguistics.

Ashish Vaswani, Noam Shazeer, Niki Parmar, Jakob Uszkoreit, Llion Jones, Aidan N Gomez, Łukasz Kaiser, and Illia Polosukhin. 2017. Attention is all you need. In Advances in Neural Information Processing Systems, pages 5998-6008.

Lu Wang and Wang Ling. 2016. Neural network-based abstract generation for opinions and arguments. In Proceedings of the 2016 Conference of the North American Chapter of the Association for Computational Linguistics: Human Language Technologies, pages 47-57, San Diego, California. Association for Computational Linguistics. 
Theresa Wilson and Janyce Wiebe. 2003. Annotating opinions in the world press. In Proceedings of the Fourth SIGdial Workshop of Discourse and Dialogue, pages 13-22.

Hong Yu and Vasileios Hatzivassiloglou. 2003. Towards answering opinion questions: Separating facts from opinions and identifying the polarity of opinion sentences. In Proceedings of the 2003 conference on Empirical methods in natural language processing, pages 129-136.

Tianyi Zhang, Varsha Kishore, Felix Wu, Kilian Q. Weinberger, and Yoav Artzi. 2020. BERTScore: Evaluating Text Generation with BERT. In International Conference on Learning Representations.

Ingrid Zukerman, Richard McConachy, and Sarah George. 2000. Using argumentation strategies in automated argument generation. In INLG'2000 Proceedings of the First International Conference on Natural Language Generation, pages 55-62, Mitzpe Ramon, Israel. Association for Computational Linguistics.

\section{A Training Details}

\section{A.1 Experiment Settings}

In this section, we describe our experiment settings in more details for reproducibility. We randomly split the MULTIOPED dataset into 70\%/10\%/20\% splits for training, validation, and testing, respectively. We train each system for 6 epochs using the same training set, and use the validation set to find the best $\alpha_{1}$ and $\alpha_{2}$. We report the test set results in Table 3. All test set results are averaged results using three different random initilizations. The approximate training time for a system trained with 6 epochs on a 12GB GPU is less than an hour.

For results shown in Table 3, we use $\alpha_{1}=30$ in + REL, $\alpha_{2}=1$ in + STANCE, and $\alpha_{1}=1, \alpha_{2}=1$ in + REL \& STANCE, as they achieved the best results in the dev set during hyperperamater tuning A.2.

\section{A.1.1 BART}

BART pre-trained model has been proved effective on text generation, question answering, and summarization tasks (Lewis et al., 2020). Given the limited size of our dataset, we finetune on BART to transfer learn from the large amount of data it was pre-trained on. We use BART base with 6 encoder and decoder layers with hidden size of 768 . We use AdamW with learning rate $3 \mathrm{e}-5$ as our optimizer.

\section{A.1.2 BERT Relevance Classifier}

BERT pre-trained model has demonstrated its power in question answering, language inference, and text classification tasks (Devlin et al., 2019). We finetune BERT-mini on PERSPECTRUM dataset first on relevance classification task (Chen et al., 2019), and then finetune it on our dataset, yielding an accuracy of $92 \%$ on evaluation set ( $20 \%$ of the data). The finetuned BERT model has 4 layers and hidden size of 256 .

\section{A.1.3 BERT Stance Classifier}

As the Relevance Classifier, we also finetune it on PERSPECTRUM dataset before training on our dataset. However, we use a larger BERT model with 8 layers and hidden size of 768 since it is a slightly more difficult task than the relevance task. For the 30 queries (2\% of the whole corpus) that are labeled as open-ended questions and do not constitute a clear stance, we exclude them from the training of BERT stance classifier. Our classifier eventually achieves $75 \%$ accuracy in the $20 \%$ evaluation set. 


\section{A.2 Hyperparameter Tuning}

To control the degree we penalize our model using auxiliary loss, we introduce hyperparameters $\alpha$. We use the $10 \%$ validation set to choose our best parameters. We tune $\alpha$ for different values from 0.1 to 50 to examine its affect on our model. We choose the best $\alpha$ according to their relevance and stance scores, and if there is a tie, we select the one with the higher ROUGE2 score. Table 7, Table 8, and Table 9 show the validation set results for tuning the BART+Rel, BART+Stance, and BART+Rel \& Stance systems, respectively.

\begin{tabular}{c|c}
\hline$\alpha_{1}$ & Relevance Score \\
\hline 0.1 & 90.0 \\
1 & 91.43 \\
5 & 88.21 \\
15 & 92.5 \\
$\mathbf{3 0}$ & $\mathbf{9 2 . 5}$ \\
50 & 92.5 \\
\hline
\end{tabular}

Table 7: Tuning $\alpha_{1}$ for BART + Rel. Here we choose $\alpha_{1}=30$ since it has the highest ROUGE2 score.

\begin{tabular}{c|c}
\hline$\alpha_{2}$ & Stance Score \\
\hline 0.1 & 72.14 \\
$\mathbf{1}$ & $\mathbf{7 2 . 5 0}$ \\
5 & 64.64 \\
15 & 64.29 \\
30 & 60.36 \\
50 & 62.50 \\
\hline
\end{tabular}

Table 8: Tuning $\alpha_{2}$ for BART + Stance

\begin{tabular}{cc|cc}
\hline$\alpha_{2}$ & $\alpha_{1}$ & Relevance & Stance \\
\hline 0.1 & 1 & 92.86 & 71.07 \\
0.1 & 5 & 88.21 & 63.93 \\
0.1 & 50 & 92.5 & 64.64 \\
$\mathbf{1}$ & $\mathbf{1}$ & $\mathbf{9 3 . 9 3}$ & 68.93 \\
1 & 5 & 90.71 & $\mathbf{7 1 . 7 9}$ \\
1 & 50 & 93.57 & 70.36 \\
\hline
\end{tabular}

Table 9: Tuning $\alpha_{1}$ and $\alpha_{2}$ for BART + Rel \& Stance. Here we choose $\left(\alpha_{1}=1, \alpha_{2}=1\right)$ over $\left(\alpha_{1}=5, \alpha_{2}=\right.$ 1) since it has a higher ROUGE2 score.

\section{A.3 Measure of Agreement}

We use Cohen's and Fleiss kappa to measure the inter-rater agreement among annotators (Fleiss and Cohen, 1973). We calculate Cohen's kappa agree- ment when there are only two raters, and Fleiss's kappa when there are more than two.

Cohen's Kappa: Let $\mathrm{n}$ be the number of instances to be labeled by A and B two raters. $\mathrm{g}$ is the number of distinct categories, and $f_{i j}$ denotes the frequency of the number of subjects with the ith categorical response for rater $A$ and the jth categorical response for rater Y. The kappa agreement is then calculated as

$$
\begin{aligned}
p_{0} & =\frac{1}{n} \sum_{i=1}^{g} f_{i i} \\
p_{e} & =\frac{1}{n^{2}} \sum_{i=1}^{g} f_{i+} f_{+i} \\
\kappa & =\frac{p_{0}-p_{e}}{1-p_{e}}
\end{aligned}
$$

where $f_{i+}$ is the total for the ith row $f_{+i}$ and is the total for the ith column in the frequency table.

Fleiss Kappa: Let $\mathrm{N}$ be the total number of subjects, let $\mathrm{n}$ be the number of ratings per subject, and let $\mathrm{k}$ be the number of categories into which assignments are made. Let $n_{i j}$ represent the number of raters who assigned the $\mathrm{i}$-th subject to the $\mathrm{j}$-th category. The kappa agreement is calculated as

$$
\begin{aligned}
p_{i} & =\frac{1}{n(n-1)} \sum_{j=1}^{k} n_{i j}\left(n_{i j}-1\right) \\
\bar{P} & =\frac{1}{N} \sum_{i=1}^{N} p_{i} \\
p_{j} & =\frac{1}{N n} \sum_{i=1}^{N} n_{i j} \\
P_{e} & =\sum_{j=1}^{k} p_{j}^{2} \\
\kappa & =\frac{\bar{P}-P_{e}}{1-P_{e}}
\end{aligned}
$$

\section{B Example Screenshots from ThePerspective Website and MTurk Annotation Interface}

In this section, we show example screenshots from the website where we extract the data, www. theperspective.com/perspectives, and example screenshots of our data annotation process.

For the three data annotation tasks using Mechanical Turk, stance annotation, implicit reference resolution, and topic annotation, we present 
the Turkers with definitions and instructions of the tasks that we require them to do, and 3-6 example questions with our expected answers. We ask them to read and comprehend our instructions before annotating, and use random control sets to filter out invalid annotations. More details can be found in the screenshots below. 


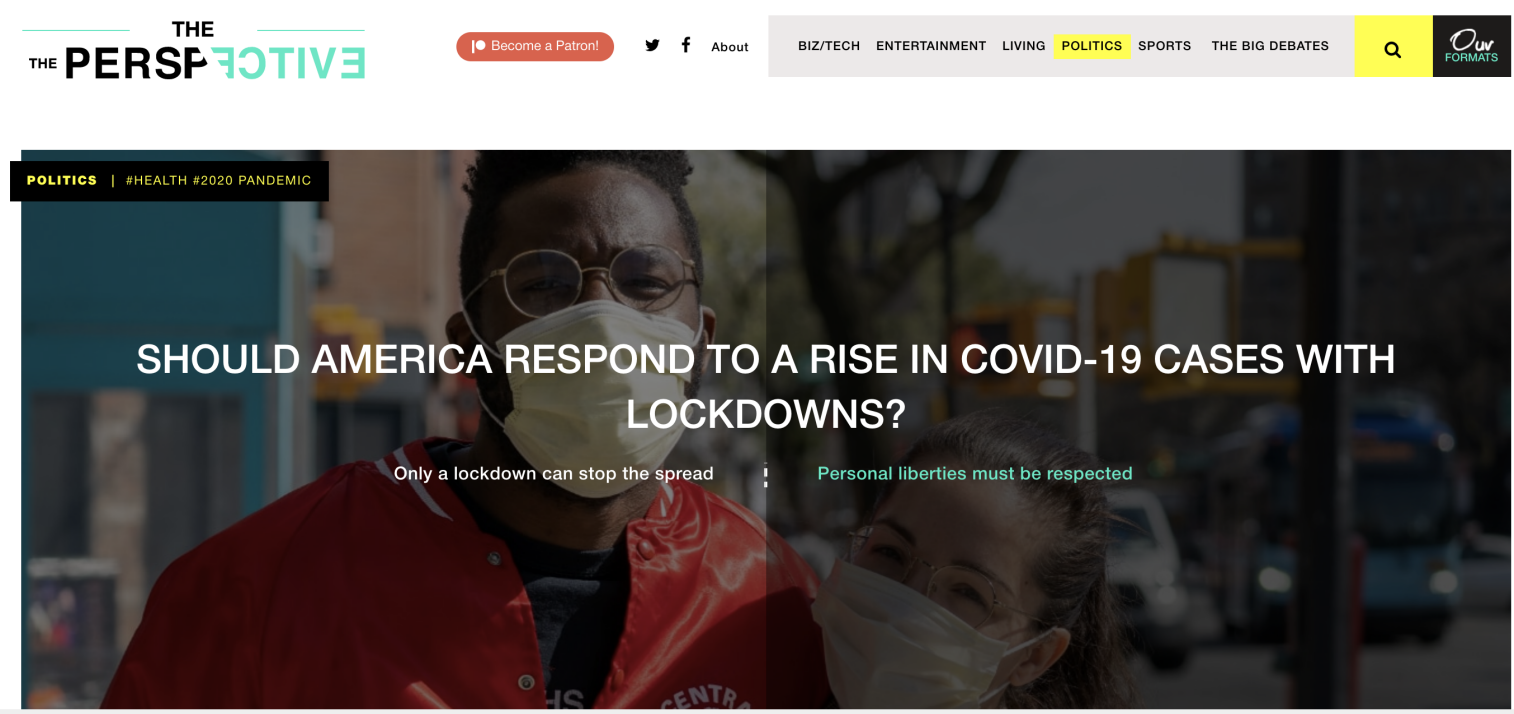

Figure 6: An example of a query and its two perspectives in ThePerspective website

Six months after the last big lockdown, it is clear that a new one is needed, believes German Lopez of Vox. Daily new cases of COVID-19 have risen as high as 180,000 , and hospitals all over the country are nearing full capacity.

Over 1,000 Americans are dying every day, which may rise to 2,000 and 3,000 before the end of the year. This fast spread has made containing the virus much harder. Past lockdowns were highly effective at stopping the virus from spreading and helped the country stabilize the infection rate. They proved to be effective in other countries as well. A new lockdown, with financial support for businesses, is direly needed to curtail further disaster.

\section{《KEEP ON READING AT VOX}

While the COVID-19 pandemic has to be taken serious, violating citizens' civil liberties is not acceptable, suggests Jonathan S. Tobing of The New York Post. The government has already gone too far regarding excessive and obstructive rules. Head-scratching measures like closing churches while leaving open casinos, which happened in Nevada, show how poorly thought out much of this strategy is. That pandemic is being politicized, as certain gatherings, such as religious ones, are cracked down upon, while Black Lives Matter protests go unpunished. We need to remember to respect individual freedoms, first and foremost, and not allow unlimited government control.

\section{KEEP ON READING AT THE NEW YORK POST>} $>$

Figure 7: An exmaple of two abstracts and their links to news editorials in ThePerspective website 


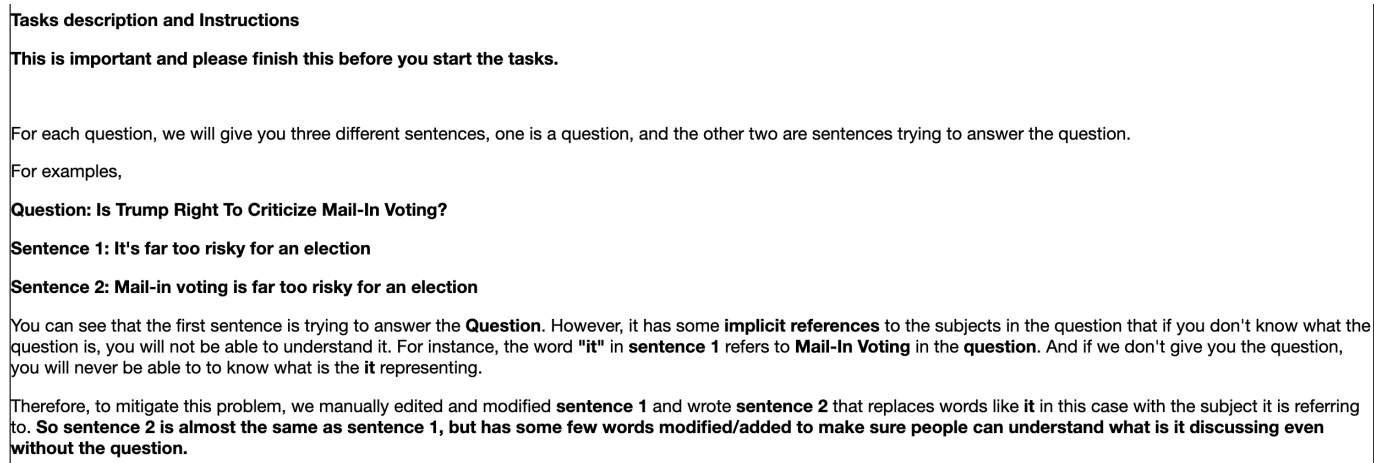

Figure 8: A screenshot of the MTurk annotation instruction for implicit reference resolution part1

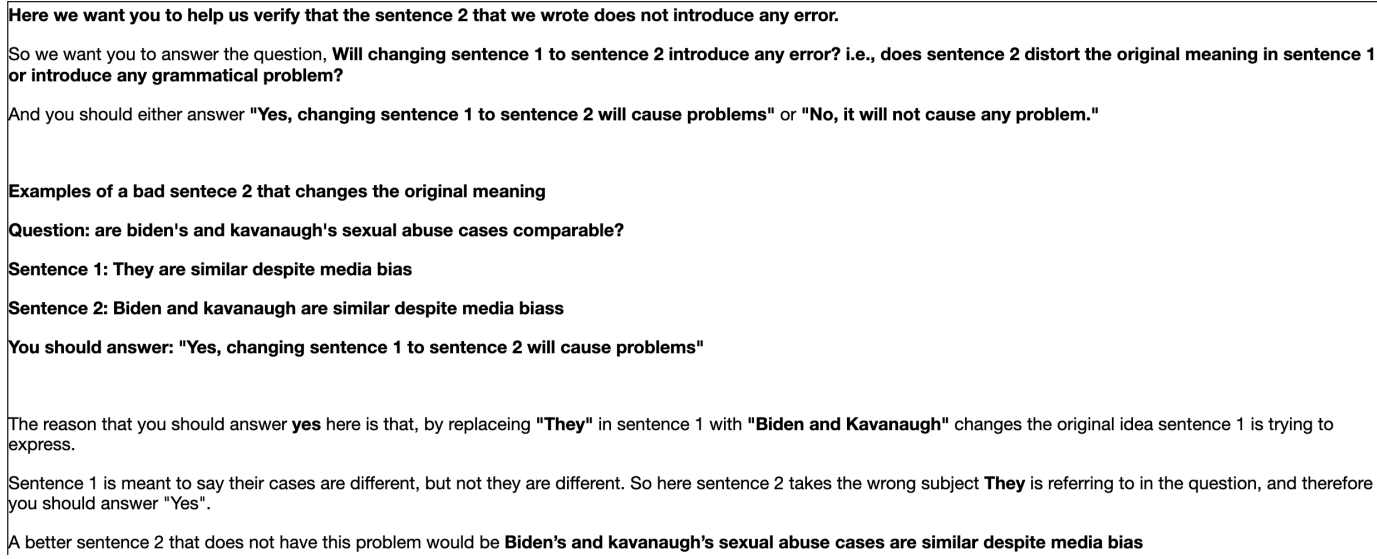

Figure 9: A screenshot of the MTurk annotation instruction for implicit reference resolution part2

Summary

Choose "Yes" if sentence 2 changes the original meaning in sentence 1 or sentence 2 has a grammatical error.

Chosse "No" if sentence 2 means exactly the same thing as sentence 1 and has no grammar error.

To better make sure that you will answer them seriously, a few examples in the dataset are set as control questions. If you can not get them correct, we will have no choice but to reject your submission. So please be patient and honest. Don't put in random selections or we will not be able to pay you. Thank you for answering the question!

Note: Make sure you have answered all the questions before you click any submit button so we can record your results.

Figure 10: A screenshot of the MTurk annotation instruction for implicit reference resolution part3

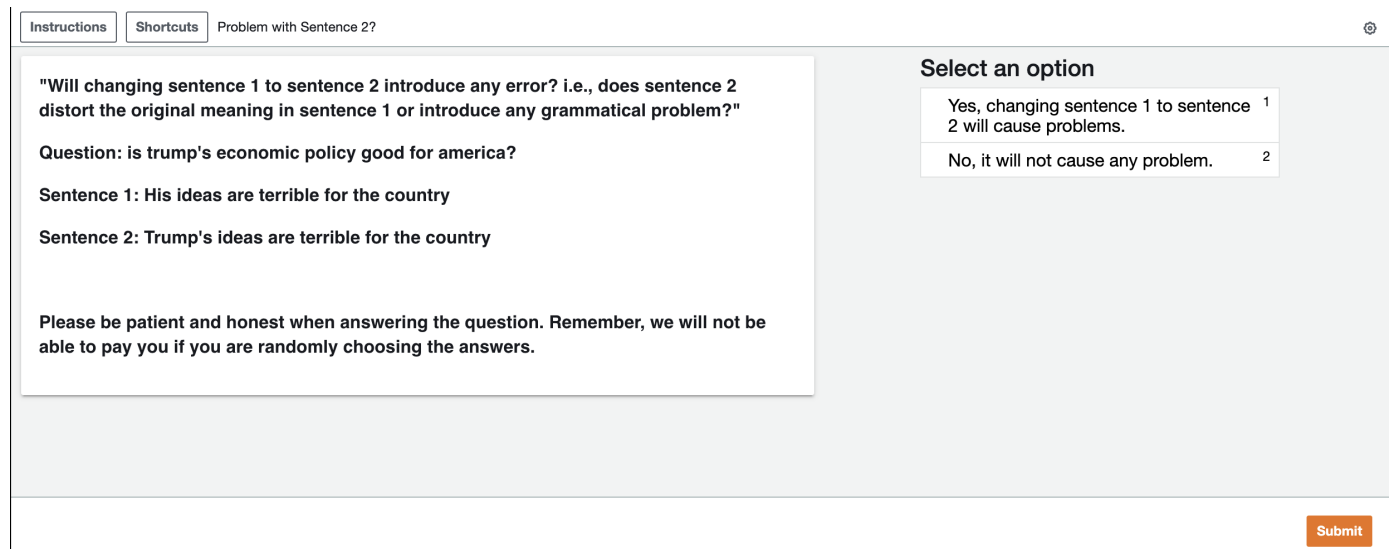

Figure 11: A screenshot of the MTurk annotation example for implicit reference resolution 


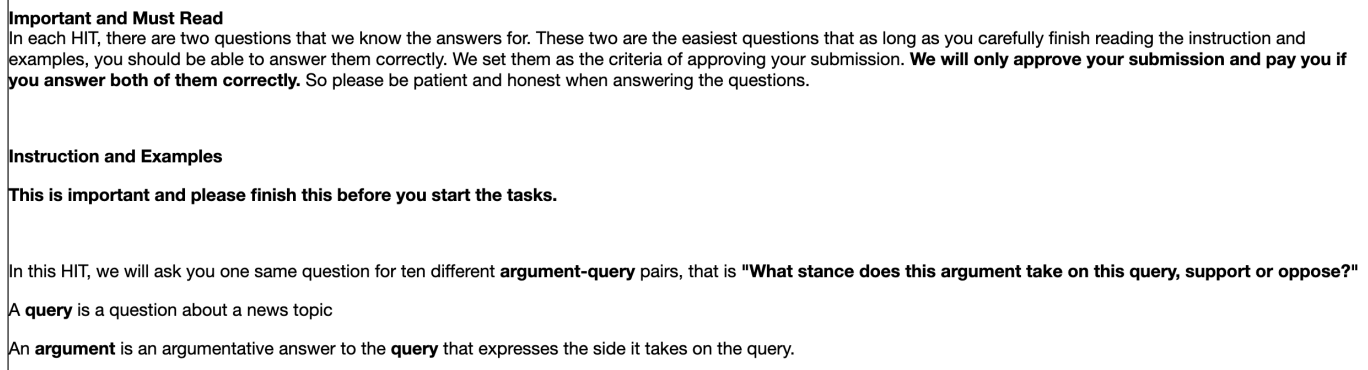

Figure 12: A screenshot of the MTurk annotation instruction for stance annotation part1

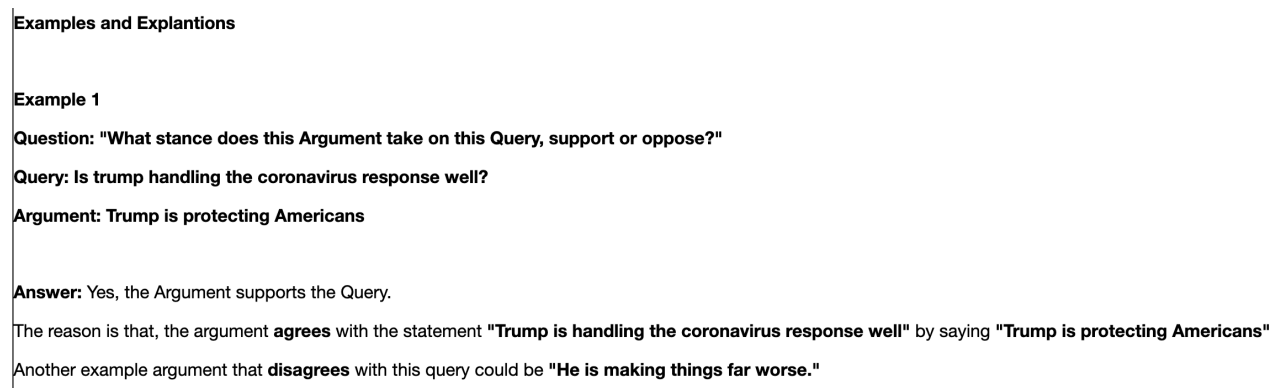

Figure 13: A screenshot of the MTurk annotation instruction for stance annotation part2

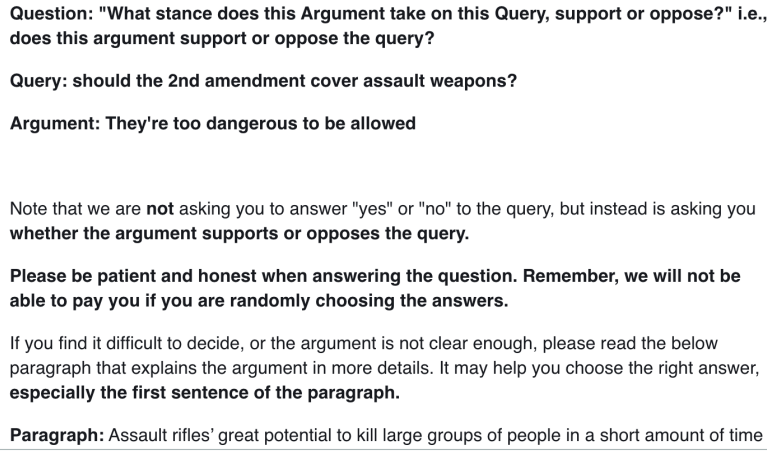

vercul all unuvi!

Yes, the argument supports the query.

No, the argument opposes the query. 2

Figure 14: A screenshot of the MTurk annotation example for stance annotation. Note that there will be more sentences shown in the Paragraph line if the user scrolls down. 
Instruction

In this HIT, we will ask you one same question for ten different sentences, that is "Which category of news topics is discussed in this sentence?" Each sentence will discuss a popular news topic in everyday life. We will need you to help us decide which category of news topic is the sentence discussing, e.g., US

Definitions and examples of the categories

This is important and please finish this before you start the tasks.

US Politics: If you see US foreign policies, US politician names, political events, or political parties, etc, you consider it belongs to the US Politics category. However, select this only if the only topic discussed in the sentence is about politics but no other categories are invovled.

Example: did president trump obstruct justice?

COVID-19: If you see it discusses anything about pandemic, covid-19/coronavirus, or lockdown, etc, you consider it belongs to the COVID-19 category

Example: should we end covid-19 security measures soon?

Figure 15: A screenshot of the MTurk annotation instruction for topic annotation part1

World News If you see it discusses anything or anyone that's outside of the United States, e.g., other countries' politics, new, etc, you consider it belongs to the World
News category.
Example: is brexit justified?
Entertainment: If you see it discusses anything about a song, a movie, a video game etc, you consider it belongs to the Entertainment category.
Example: is a star is born worth all the hype?
Sports: If you see it discusses anything about a type of sport, a sport's league, or a sport player, etc, you consider it belongs to the Sports category.
Example: who will win the 2018 nba playoffs?
Gender Equality: If you see it discusses anything about feminism, gender quality, equal pay, etc, you consider it belongs to the Gender Equality category.
Example: are conservatives using feminism to their own advantage?
Economy: If you see it discusses anything about a country's economy, a trade war, business, companies, anything about money, etc, you consider it belongs to the
Economy category.

Figure 16: A screenshot of the MTurk annotation instruction for topic annotation part2

Racial Equality: If you see it discusses anything about black voices, racial discrimination, racial equality, etc, you consider it belongs to the Racial Equality category.
Example: was the starbucks arrest racially motivated?
Other Topics If you see it discusses anything about topics other than the ones above, you consider it belongs to the Other Topics category.
Example: was elon musk's space x project justified?
If you think that it is discussing topics in more than one categories, e.g., it discusses topics in both US Politics and coviD-19, choose the one that you think it is
emphasizing more and is the key point that it is discussing.. Think about which category will this news headlines appear on CNN, Fox News, NBC, etc.
Example 1
Question: "Which category of news topics is discussed in this sentence?"
Query: Is trump handling the coronavirus response well?
Answer: COVID-19
We see that here it discusses topics both in us Politics and in coviD-19. However, we believe that the emphasis is more on coVID-19 because it only mentions one
bolitician name. but the kev subiect that it is discussina is COVID-19. TVDically. when vou see a sentence askina how a oolitician does on the matter of COVID-19. vou should

Figure 17: A screenshot of the MTurk annotation instruction for topic annotation part3

\begin{tabular}{l|l|l|}
$\begin{array}{l}\text { Question 1: Which category of news topics is discussed in this sentence? } \\
\text { Sentence: should trump choose the new cfpb head? }\end{array}$ & $\begin{array}{l}\text { Select an option } \\
\text { US Politics }\end{array}$ \\
\hline $\begin{array}{l}\text { Please be patient and honest when answering the question. Remember, we will not be } \\
\text { able to pay you if you are randomly choosing the answers. }\end{array}$ & COVID-19 2 \\
\hline & World News & 3 \\
\hline & Entertainment & 4 \\
\hline & Sports & 5 \\
\hline Gender Equality & 6 \\
\hline
\end{tabular}

Figure 18: A screenshot of the MTurk annotation example for topic annotation 